\section{Using an Intensified Infection Prevention Intervention to Control Carbapenemase- Producing Enterobacteriaceae at a Thai Center}

To the Editor-Emergence of carbapenemase-producing Enterobacteriaceae (CPE) in several parts of the world has heightened efforts to limit the transmission of these microorganisms. ${ }^{1.4}$ Controlling CPE can be difficult in resource-limited settings because of generally higher nurse-to-patient ratios and less available infection prevention resources in developing countries. We report our experience using an intensified infection prevention intervention to control the transmission of CPE in a Thai medical center.

At Thammasat University Hospital, since January 2011, any phenotypically CPE isolate is submitted for testing to detect important carbapenemase genes $\left(\mathrm{bla}_{K P O} \mathrm{bla}_{N D M}, \mathrm{bla}_{I M P}\right)$ according to a previously described molecular method. ${ }^{5,6}$ The hospital epidemiologist is immediately notified, the index patient is isolated in a single room, and infection prevention intervention is promptly implemented. Infection prevention measures include (1) implementation of enhanced contact isolation precautions (ie, strict adherence to hand hygiene protocols before and after patient care and use of gowns and gloves for patient care of index cases), (2) performance of active surveillance cultures of all patients in the index units for $\mathrm{CPE}$, (3) environmental cleaning with detergents, and with phenolic agents for surfaces contaminated with body fluids or with blood, (4) up-to-date education programs for healthcare personnel, and (5) delivery of unit-specific feedback and real-time feedback to healthcare workers on adherence to infection prevention measures. If there are 2 or more cases, a cohort area is created.

In this study, case patients were defined as patients with nosocomial colonization and/or infection with CPE identified by clinical cultures more than $48 \mathrm{~h}$ after admission to the study unit. Nosocomial acquisition of CPE was defined as detection of this microorganism by active surveillance culture more than $48 \mathrm{~h}$ after admission, when initial admission active surveillance cultures yielded negative results. Active surveillance cultures for $\mathrm{CPE}$ were performed on tracheal aspirate specimens and rectal swab specimens (if the initial tracheal aspirate specimens were negative) on day 0 , day 7 , and every week until discharge from this unit for all patients in the unit. Hand hygiene adherence, as well as adherence to infection prevention measures at the month prior to admission of the 3 index units is shown in Table 1.

From January 1, 2011, to April 25, 2012, there were 3 suspected cases of CPE (2 Klebsiella pneumoniae and $1 \mathrm{Kleb}$ siella oxytoca) that occurred in 3 medicine wards. These were 30 -bed units, with nurse-to-patient ratios of about $1: 8$. One of the suspected cases carried the NDM-1 gene, whereas the other 2 cases had no identifiable carbapenemase-producing genes from our test panel. All 3 cases were referred by outside hospitals. After the interventions, adherence to hand hygiene before and after patient contact as well as to contact isolation precautions for all 3 units significantly improved (Table 1). There were no additional cases of infection, colonization, or transmission identified in the 3 index units. A total of 336 surveillance cultures were performed on 78 patients during the 41 cumulative hospital-days the case patients were admitted. The total costs of the interventions in 3 units are US $\$ 3,360$ inclusive of molecular testing for carbapenemase genes and active surveillance cultures.

Our study suggests that $\mathrm{CPE}$ is emerging in Thailand, and intensified infection prevention programs are needed to control index cases because intensive interventions at the early stage may abort hospital outbreaks. If this outbreak had been detected later, after the organism had become widespread in

TABLE 1. Adherence to Hand Hygiene and Infection Prevention Measures at 3 Index Units

\begin{tabular}{|c|c|c|c|}
\hline Variables & $\begin{array}{c}\text { Unit } 1 \\
\text { (index case 1) }\end{array}$ & $\begin{array}{c}\text { Unit } 2 \\
\text { (index case 2) }\end{array}$ & $\begin{array}{c}\text { Unit } 3 \\
\text { (index case } 3 \text { ) }\end{array}$ \\
\hline \multicolumn{4}{|l|}{ Hand hygiene adherence level $(\%)$ before and after patient contact ${ }^{a}$} \\
\hline 1 month before implementation of IIC program $(n=60)$ & 50 & 53 & 58 \\
\hline After intensified IIC program $(n=60)$ & $87^{\mathrm{b}}$ & $92^{\mathrm{b}}$ & $95^{\mathrm{b}}$ \\
\hline \multicolumn{4}{|l|}{ Adherence level $(\%)$ to contact isolation precautions $s^{c}$} \\
\hline 1 month before implementation of IIC program $(n=60)$ & 25 & 33 & 25 \\
\hline After intensified IIC program $(n=60)$ & $70^{\mathrm{b}}$ & $75^{\mathrm{b}}$ & $83^{b}$ \\
\hline
\end{tabular}

NOTE. IIC $=$ intensified infection prevention.

${ }^{a}$ Defined as the no. of observations confirming adherence to hand hygiene requirements divided by the total number of observations.

b $P<.001$.

c Defined as the no. of observations confirming adherence to contact isolation precaution requirements divided by the total number of observations. 
the unit and after the environment had become heavily contaminated, prevention of CPE infection and colonization/acquisition would have been more difficult or even impossible. Because interhospital transfers may serve as a source of transmission, this study illustrates the importance of additional screening strategy to detect CPE at admission, as well as a surveillance strategy for those cases at the community level after discharge to help with early containment of these pathogens.

\section{ACKNOWLEDGMENTS}

Financial support. This study was supported by the National Research University Project of the Thailand Office of Higher Education Commission (to A.A. and T.K.), Siriraj Research Development Foundation (P.K.), and Infectious Diseases and Infection Control Research Unit (to A.A. and T.K.).

Potential conflicts of interest. All authors report no conflicts of interest relevant to this article. All authors submitted the ICMJE Form for Disclosure of Potential Conflicts of Interest, and the conflicts that the editors consider relevant to this article are disclosed here.

Anucha Apisarnthanarak, $\mathrm{MD}{ }^{1}$ Pattarachai Kiratisin, $\mathrm{MD}, \mathrm{PhD}{ }^{2}$ Thana Khawcharoenporn, MD, MSc; David K. Warren, MD $^{3}$

Affiliations: 1. Division of Infectious Diseases, Thammasat University Hospital, Pratumthani, Thailand; 2. Department of Microbiology, Faculty of Medicine, Siriraj Hospital, Bangkok, Thailand; 3. Division of Infectious Diseases, Washington University School of Medicine, Saint Louis, Missouri.

Address correspondence to Anucha Apisarnthanarak, MD, Division of Infectious Diseases, Faculty of Medicine, Thammasat University Hospital, Prathumthani, Thailand, 12120 (anapisarn@yahoo.com).

Infect Control Hosp Epidemiol 2012;33(9):960-961

(1) 2012 by The Society for Healthcare Epidemiology of America. All rights reserved. 0899-823X/2012/3309-0018\$15.00. DOI: $10.1086 / 667386$

\section{REFERENCES}

1. Shakil S, Azhar EI, Tabrez S, et al. New Delhi metallo- $\beta$-lactamase (NDM-1): an update. I Chemother 2011;23:263-265.

2. Nordmann P, Poirel L, Walsh TR, Livermore DM. The emerging NDM carbapenemase. Trends Microbiol 2011;19:588-595.

3. Cornaglia G, Giamarellou $H$, Rossolini GM. Metallo- $\beta$-lactamases: a last frontier for $\beta$-lactams? Lancet Infect Dis 2011;11: 381-393.

4. Walsh TR. Emerging carbapenemase: a global prospective. Int $J$ Antimicrob Agents 2010;36:S8-S14.

5. Kumaramy KK, Tolema MA, Walsh TR, et al. Emergence of a new antibiotic resistance mechanism in India, Pakisatan, and the UK: a molecular, biological, and epidemiological study. Lancet Infect Dis 2010;10:597-602.

6. Ellington MJ, Kistler J, Livermore DM, Woodward, N. Mutiplex PCR for rapid detection of genes encoding acquired metallo- $\beta$ lactamases. I Antimicrob Chemother 2007;59:321-322.

\section{Chlorhexidine Is Better than Aqueous Povidone Iodine as Skin Antiseptic for Preventing Surgical Site Infections}

To the Editor-I have read with great interest the article by Kamel et $\mathrm{al}^{1}$ in Infection Control and Hospital Epidemiology. The authors have conducted a systematic review on what is described as comparison of preoperative skin antisepsis preparations for preventing surgical site infections (SSIs). On the basis of their analyses, the authors conclude that "given the heterogeneity of the studies and the results, conclusions about which antiseptic is more effective at reducing SSIs cannot be drawn." However, I believe that this conclusion may be tempered by the studies included.

In many countries the most common antiseptic agents used for skin preparation before surgery are povidone iodine and chlorhexidine. Both compounds are available in aqueous formulations and in alcoholic formulations. The use of one antiseptic agent over another depends on the choice of the surgeon rather than national recommendations. However, studies selected by Kamel et al ${ }^{1}$ may help in choosing the best agent.

Among the 9 studies included in their analysis, the 3 randomized controlled trials comparing aqueous $(n=1)$ or alcoholic $(n=2)$ formulations of chlorhexidine to aqueous povidone iodine in a total of 1,599 patients reported lower SSI rates with chlorhexidine. The 2 cohort studies led to conflicting results. However, their conclusions need to be tempered, given the inherent limitations to the lack of random assignment, particularly the inadequate control of major confounders. Moreover, the cohort study in favor of the use of povidone iodine was unable to demonstrate a significant reduction of SSIs in the multivariate analysis (odds ratio [OR], 1.35 [95\% confidence interval [CI], 0.97-1.87]; $P=$ .073 ) despite the inclusion of 3,135 patients, yet being by far the study with the largest sample size. ${ }^{2}$ Finally, the last 4 studies were not direct comparisons of chlorhexidine-based formulations to povidone iodine-based formulations.

The superiority of chlorhexidine on povidone iodine was confirmed by 2 meta-analyses published in the British Journal of Surgery and in Infection Control and Hospital Epidemiology in 2010. The meta-analysis of Noorani et a ${ }^{3}$ included 6 studies published between 1988 and 2010 and a total of 5,031 patients. The use of chlorhexidine was associated with a risk reduction of SSIs compared with povidone iodine (OR, 0.68 [95\% CI, 0.50-0.94]; $P=.019$ ). The meta-analysis of Lee et $\mathrm{al}^{4}$ included 7 randomized studies and a total of $3,437 \mathrm{pa}-$ tients. Similarly, the use of chlorhexidine reduced the risk of SSIs compared to povidone iodine (OR, $0.64[95 \% \mathrm{CI}$, $0.51-0.80$ ]; $P<.0001$ ). The results of these 2 meta-analyses are concordant, which is not surprising because 4 studies and 2,952 patients were included in both meta-analyses, including 